\title{
El aprendizaje cooperativo mejora el rendimiento académico The cooperative learning improves the academic performance
}

\author{
Sonia Lorente ${ }^{1,2}$, Jordi Fauquet ${ }^{1,3}$, Diego Redolar ${ }^{1,4}$, Genís Prat-Ortega $^{1,5}$, Alfredo Pardo ${ }^{1}$, Albert Bonillo ${ }^{1}$ \\ sonia.lorente@uab.cat,jordi.fauquet@uab.cat,diego.redolar@uab.cat,genis.prat@uab.cat, \\ alfredo.pardo@uab.cat, albert.bonillo@uab.cat
}

\author{
${ }^{1}$ Departamento de Psicobiología y Metodología en \\ Ciencias de la Salud. Universidad Autónoma de \\ Barcelona, UAB. España. \\ ${ }^{2}$ Ámbito Materno-Infantil, Consorci Sanitari de \\ Terrassa. España \\ ${ }^{5}$ Institut d'Investigacions Biomèdiques August Pi i \\ Sunyer (IDIBAPS)
}

\author{
${ }^{3}$ Neuroimaging Research Group (NRG). \\ Neurosciences Research Programme, Hospital del \\ Mar Medical Research Institute (IMIM) \\ ${ }^{4}$ Cognitive NeuroLab, Faculty of Psychology and \\ Educational Sciences, Universitat Oberta de \\ Catalunya (UOC)
}

Resumen. Introducción. El aprendizaje cooperativo emerge como una metodología innovadora adaptada a las propuestas de mejora del Espacio Europeo de Educación Superior (EEES). Objetivo. Mejorar el rendimiento académico en estudiantes universitarios incorporando estrategias de aprendizaje cooperativo facilitadoras del trabajo en equipo. Metodología. Evaluamos el rendimiento académico y las competencias de trabajo en equipo mediante el Cuestionario de Evaluación de Aprendizaje Cooperativo (CAC). Para índices descriptivos, correlaciones y fiabilidad, se utilizó SPSS 20.0 (IBM, Chicago, IL), y para el análisis factorial confirmatorio (AFC) se utilizó Mplus 8.4. Resultados. El rendimiento académico se incrementó considerablemente respecto al curso anterior. Del total de 411 estudiantes, $\mathrm{n}=114$ cumplimentaron el $\mathrm{CAC}$, con puntuaciones en Habilidades Sociales $M=4,2$ (SD 0,7), Procesamiento Grupal $M=4,3$ (SD 0,9), Interdependencia Positiva $\mathrm{M}=4,0$ (SD 0,7), Interacción Promotora $\mathrm{M}=3,8$ (SD 1,0), y Responsabilidad Individual $\mathrm{M}=4,3$ (SD 0,6). El AFC muestra que las escalas de Habilidades Sociales y Procesamiento Grupal están altamente correlacionadas, derivando en un modelo de cuatro dimensiones, a diferencia de la validación original, con cinco subescalas. Conclusiones. La implementación de este método mejora el rendimiento académico y las competencias de trabajo en equipo, pero sería necesario validar este cuestionario en otros contextos universitarios para confirmar nuestros resultados.

\section{Palabras clave. Aprendizaje cooperativo, rendimiento académico,} competencias, enseñanza universitaria

Abstract. Introduction. Cooperative learning emerges as an innovative methodology adapted to the proposals for improvement of the European Higher Education Area (EHEA). Objective. Improving the academic performance in university students incorporating cooperative learning strategies that facilitate the teamwork. Methodology. We assessed the academic performance and teamwork skills using the Cooperative Learning Assessment Questionnaire (CAC). For descriptive indices, correlations, and reliability, SPSS 20.0 (IBM, Chicago, IL) was used, and for confirmatory factor analysis (CFA), Mplus 8.4. Results. The academic performance was considerably improved compared to the last academic course. Of the total of 411 students, $n=114$ completed the CAC, with scores in Social Skills $\mathrm{M}=4.2$ (SD 0.7), Group Processing $\mathrm{M}=4.3$ (SD 0.9), Positive Interdependence $\mathrm{M}=4,0$ (SD 0.7), Promoter Interaction $\mathrm{M}=3.8$ (SD
1.0), and Individual Responsibility $\mathrm{M}=4.3$ (SD 0.6). The CFA shows that the Social Skills and Group Processing scales are highly correlated, resulting in a four-dimensional model, unlike the original validation, with five subscales. Conclusions. The implementation of this method improves the academic performance and teamwork skills, but it would be necessary to validate this questionnaire in other university contexts to confirm our results.

\section{Keywords: Cooperative learning, academic performance, competencies, higher education}

\section{INTRODUCCIÓN}

El Espacio Europeo de Educación Superior (EEES) propone la mejora de la calidad de la enseñanza fomentando el protagonismo del estudiantado y el aprendizaje activo. Las nuevas metodologías estarían dirigidas a favorecer escenarios que faciliten tanto el aprendizaje autónomo, autorregulado y eficaz, vinculado a las teorías socio-constructivista, como las competencias genéricas y específicas vinculadas a las titulaciones universitarias (Domingo, 2010; Guerra et al., 2019). En este contexto, el aprendizaje cooperativo emerge como una metodología innovadora que se adapta a las propuestas de mejora de la calidad de la enseñanza. El aprendizaje cooperativo consta de cinco elementos esenciales: (a) Interdependencia positiva, que tiene lugar cuando los resultados del trabajo depende de todos los componentes, de forma que la ejecución y la producción son conjuntas, y la calificación final es igual para todos los integrantes; (b) Interacción positiva cara a cara cuando los integrantes del grupo se explican verbalmente como resolver un problema o una duda; (c) Responsabilidad individual, evaluada por el docente cuando hace exámenes o bien hace una elección al azar de uno de los estudiantes para presentar resultados de un trabajo; (d) Habilidades cooperativas y liderazgo, gestión de conflictos, que a veces pueden precisar apoyo por parte del docente, como una habilidad académica más dentro de su proceso de aprendizaje; y, (e) Auto-análisis de grupo, reflejada en el buen funcionamiento del grupo, incluyendo autorreflexión sobre lo 
aprendido, de acuerdo con las teorías constructivistas. El aprendizaje cooperativo promueve, pues, la comunicación entre los componentes del grupo y su grado de implicación en las tareas propias del curso, fomentando la autonomía y la responsabilidad, constituyendo así una metodología adecuada para mejorar el aprendizaje y consecuentemente el rendimiento académico (Gillies, 2014; Johnson \& Johnson, 2014; Martínez et al., 2011; Valero-García, 2017)

\section{CONTEXTO}

En este contexto de mejora de la calidad de la enseñanza y del rendimiento académico nos planteamos este proyecto de innovación en la asignatura "Análisis de datos", impartida en segundo curso del Grado de Psicología de la Universidad Autónoma de Barcelona (UAB) durante el curso académico 2020-2021. En este entorno universitario, nos planteamos como objetivo principal la mejora del rendimiento académico implementando una metodología de aprendizaje cooperativo ajustada a las directrices del Espacio Europeo de Educación Superior. En este marco, se derivan los siguientes objetivos específicos:

1. Fomentar la actividad de trabajo autónomo no supervisado de acuerdo las directrices de la guía docente.

2. Fomentar la asistencia del alumnado a los seminarios para la resolución de dudas.

3. Fomentar la interdependencia positiva y la responsabilidad individual del alumnado.

4. Favorecer el aprendizaje fomentando la interdependencia positiva y la responsabilidad individual.

5. Mejorar los resultados de aprendizaje, tanto en relación a las evidencias grupales como individuales.

6. Validación estructural del Cuestionario de Evaluación de Aprendizaje Cooperativo (CAC) en contexto universitario (Fernández-Rio et al., 2017)

\section{DESCRIPCIÓN}

\section{A. Metodología y Plan de Trabajo}

Para facilitar la implementación de la metodología de aprendizaje cooperativo diseñamos dos pruebas de evaluación práctica consistentes en la resolución de un caso de investigación por un equipo de trabajo de cinco miembros que se conformaron aleatoriamente. La evaluación de esta prueba se realizó mediante la exposición oral de los resultados por uno de los miembros del equipo, escogido por el docente al azar, justo antes de la presentación. La nota obtenida era la misma para todos los miembros del equipo, y la calificación se realizó siguiendo una rúbrica, disponible tanto para los docentes como para los equipos de trabajo. La rúbrica puntuaba diferentes aspectos de la exposición oral, como la defensa y los aspectos formales, constituyendo un $75 \%$ de total de la nota, así como el contenido escrito de la prueba, constituyendo el $25 \%$ restante. Tanto la metodología de exposición grupal como la asignación de la nota a todos los miembros por igual favorecerían la interdependencia y el aprendizaje cooperativo. Atendiendo a lo anterior, se diseñó un plan de trabajo estructurado en cinco fases.

Fase 1. Antes de iniciar el periodo de clases, se procedió a la adecuación del material destinado al trabajo autónomo, consistente en seis casos prácticos diseñados para realizar en equipo, también denominados dossiers de prácticas. Al inicio del periodo lectivo se formaron los equipos por asignación aleatoria y se les asignó un nombre para poder identificarlos correctamente.

Fase 2. Durante el periodo lectivo, se realizaron seis seminarios, uno por semana, destinados a la resolución de dudas derivadas de la resolución de los casos prácticos. Dada la situación de excepcionalidad derivada de la COVID19, parte de estos seminarios se realizaron de forma virtual (Microsoft Teams).

Fase 3. Realización de dos pruebas de evaluación práctica que abarcaban la totalidad del temario presentado y los casos prácticos. Cada prueba práctica abarcaba el temario de tres seminarios prácticos. Dada la situación de excepcionalidad derivada de la COVID19 la primera prueba se realizó de forma virtual (Microsoft Teams), y la segunda de forma presencial.

Fase 4. Administración del cuestionario de evaluación de aprendizaje cooperativo (CAC) (Fernández-Rio et al, 2017) en el último seminario del semestre académico, que tuvo lugar en formato virtual.

Fase 5. Finalizado el semestre académico, se procedió al análisis de los datos.

\section{Cuestionario de evaluación de aprendizaje cooperativo}

La revisión de la literatura disponible sobre el tema permitió identificar diferentes instrumentos diseñados y validados para evaluar el aprendizaje cooperativo, entre otros: el Cooperative Learning Observational Schedule (Veenman et al., 2002), la Escala de Aplicación del Aprendizaje Cooperativo (CLAS) (Atxurra et al., 2015)el Cuestionario de Análisis de la Cooperación en Educación Superior (García et al., 2012), el Autoinforme de Interacción Grupal (Ibarra \& Rodríguez, 2007) para contexto universitario, y el Cuestionario de Evaluación de Aprendizaje Cooperativo (CAC) (Fernández-Rio et al., 2017) para contexto de educación secundaria y bachillerato. Dado que el cuestionario Cooperative Learning Observational Schedule se diseñó para evaluar el aprendizaje cooperativo por un observador ajeno al equipo, como el profesor, el CLAS no valoraba la competencia de Responsabilidad individual, y los instrumentos Cuestionario de Análisis de la Cooperación en Educación Superior y el Autoinforme de Interacción Grupal están entrados en la interacción de grupo, decidimos aplicar el cuestionario CAC evaluando así su validez estructural en entorno universitario.

El cuestionario de evaluación de aprendizaje cooperativo (CAC) (Fernández-Rio et al, 2017) se diseña y se valida para entornos de educación secundaria, y está estructurado en cinco dimensiones: Habilidades Sociales (4 ítems), Procesamiento grupal (4 ítems), Interdependencia Positiva (4 ítems), Interacción Promotora (4 ítems) y Responsabilidad Individual (4 ítems) con formato de respuesta en escala Likert de 5 puntos ( 1 = Totalmente en desacuerdo, $5=$ Totalmente de acuerdo). Tanto el análisis factorial de primer orden en la muestra original 
como el análisis segundo orden en el denominado Factor de Cooperación presentaron índices de ajuste adecuados.

\section{B. Análisis estadístico}

El análisis estadístico se realizó en dos fases. Para el cálculo de índices descriptivos, correlaciones -entre ítems y entre escalas- y la fiabilidad, se utilizó SPSS versión 20.0 (IBM, Chicago, IL), y para el análisis factorial confirmatorio (AFC) se utilizó Mplus 8.4. Se estimó la estructura original del CAC con cinco sub-escalas o factores latentes -que se obtienen a partir de cuatro variables observables o patentes, así como un factor de segundo nivel denominado Factor de Cooperación.

El coeficiente de curtosis multivariado de Mardia (1974) mostró una distribución -multivariante- no-normal de los ítems del CAC (coeficiente de Mardia $=527,49, \chi_{\mathrm{gl}=1}^{2}=247,9$, $\mathrm{p}<0,001)$. Se optó pues por el uso del método de estimación MLM (máxima verosimilitud con errores estándar robustos) que es robusto frente a violaciones de la normalidad (Byrne, 2012).

Por tratarse de un AFC, los índices de ajuste empleados fueron el estadístico Satorra-Bentler (S-B $\chi 2$; Satorra y Bentler, 1994), el CFI (Comparative Fit Index), el RMSEA (Root Mean Square Error Aproximation) y el SRMR (Standardized Root Mean Square Residual). Para el CFI, Bentler (1990) sugirió que los valores de 0,95 o superiores indican un buen ajuste, aunque otros investigadores han sugerido un límite en 90 (por ejemplo, Jöreskog, Sörbom, du Toit y du Toit, 2000). Para el RMSEA, los valores inferiores a 0,05 indican un ajuste excelente, y los valores inferiores a 0,08 indican solo un ajuste adecuado. Para el SRMR, Hu y Bentler (1999) sugieren que valores inferiores a 0,08 indican un buen ajuste. Se descartó el realizar un análisis de invariancia debido al reducido número de hombres participantes.

\section{Aspectos Éticos}

Atendiendo a la Instrucción 10/2020 sobre la protección de datos en materia de evaluación virtual, e informando previamente al alumnado, se grabaron las sesiones virtuales.

Para utilizar el cuestionario CAC se obtuvo autorización del autor principal. La aplicación del cuestionario se realizó mediante un formulario online (Google Forms), manteniendo el anonimato de los participantes e informando sobre el objeto del estudio, así como de la normativa de protección de datos. Todos los participantes que cumplimentaron este cuestionario firmaron el consentimiento para tratar los datos.

\section{Resultados}

\section{A. Participantes}

La matrícula de esta asignatura durante este curso académico comprendió un total de 411 estudiantes entre los cuáles $n=342$ (83\%) eran mujeres, y $n=69(19 \%)$ eran hombres, con edad media de 21,8 años (DE= 1,6 años). El total de los 411 estudiantes participaron en el proyecto de innovación docente, $\mathrm{y}$ un total de $\mathrm{n}=114(27,8 \%)$ respondieron el cuestionario de aprendizaje cooperativo.

\section{B. Indicadores}

La evaluación del impacto de la intervención procedió a partir de la información aportada por diferentes indicadores.

Para conseguir el objetivo de fomentar la actividad de trabajo autónomo se estableció la obligatoriedad de entregar como mínimo un dossier de prácticas por cada uno de los equipos. La elaboración y entrega de un dossier de prácticas suponía cumplir al menos un tercio de horas de trabajo autónomo. Esta entrega no tenía carácter evaluativo, pero se consideraba necesaria para poder presentarse a la prueba de evaluación práctica. La totalidad de los equipos entregaron un caso antes de cada evidencia, cumpliendo así este objetivo.

Con relación al objetivo de fomentar la asistencia a los seminarios, nos planteamos que sería exitoso si al menos un $85 \%$ del alumnado asistía a los seminarios. La asistencia global fue aproximadamente un 50\%. Consideramos, en este caso, que la situación de excepcionalidad pudo influir en la asistencia dificultando el cumplimiento de este objetivo.

Respecto al objetivo de fomentar la interdependencia positiva y la responsabilidad individual, así como el resto de competencias relacionadas con el aprendizaje cooperativo, consideramos que se cumpliría de forma exitosa si las puntuaciones en el cuestionario CAC eran adecuadas (puntuaciones $>4$ ).

La Tabla 1 muestra las puntuaciones por dimensión (media y desviación estándar), así como las correlaciones entre escalas, e índices de consistencia interna. Los resultados muestran puntuaciones adecuadas en cada una de las dimensiones, inclusive superiores a las puntuaciones medias de la muestra original, correlaciones con valores comprendidos entre $r=0,44$ y $r=0,91$, e índices de consistencia interna $\alpha>0.6$.

Tabla 1. Puntuaciones, correlaciones y consistencia interna.

\begin{tabular}{lccccccc}
\hline & $\mathrm{M}$ & $\mathrm{DE}$ & $1 . \mathrm{HS}$ & 2.PG & 3.IP & 4.IPR & $\alpha$ \\
\hline 1.HS & 4,2 & 0,7 & & & & & 0,86 \\
2.PG & 4,3 & 0,9 & 0,91 & & & & 0,91 \\
3.IP & 4,0 & 0,7 & 0,64 & 0,66 & & & 0,69 \\
4.IPR & 3,8 & 1,0 & 0,71 & 0,88 & 0,75 & & 0,89 \\
5.RI & 4,3 & 0,6 & 0,44 & 0,44 & 0,54 & 0,50 & 0,67 \\
\hline
\end{tabular}

Nota: $\quad H S=$ Habilidades sociales; $\quad P G=$ Procesamiento grupal; $\mathrm{IP}=$ Interdependencia positiva; $\mathrm{IPR}=$ Interacción promotora; $\mathrm{RI}=$ Responsabilidad social; $\mathrm{M}=$ Media; $\mathrm{DE}=$ Desviación Estándar; $\alpha=$ Alfa de Cronbach, consistencia interna.

Además, en el marco de este mismo objetivo, incorporamos cinco preguntas de elaboración propia para conocer la opinión del alumnado con relación al trabajo en equipo y los seminarios. Cuatro de las preguntas se elaboraron con formato de respuesta escala Likert de 5 puntos $(1=$ Totalmente en desacuerdo, $5=$ Totalmente de acuerdo), y una pregunta se elaboró con respuesta abierta para valorar opiniones. La Tabla 2 muestra los resultados de las preguntas con respuesta con escala Likert, expresados en medias y desviación estándar. 
Tabla 2. Valoración sobre trabajo en equipo y seminarios.

\begin{tabular}{lcc}
\hline & M & SD \\
\hline Trabajar en equipo ha sido una experiencia positiva & 3,9 & 1,2 \\
Trabajar en equipo favorece el aprendizaje de la materia & 4,1 & 0,9 \\
Los seminarios facilitan la comprensión de la materia & 4,6 & 0,6 \\
$\begin{array}{l}\text { Entregar casos prácticos facilita la comprensión de la } \\
\text { materia }\end{array}$ & 4,0 & 1,0
\end{tabular}

Nota: $\mathrm{M}=$ Media; $\mathrm{SD}=$ Desviación estándar.

De los 114 participantes, un total de 25 respondieron la pregunta con respuesta abierta, de los cuales $n=14(12,3 \%)$ manifestaron su desacuerdo con la confección de los grupos de forma aleatoria, pues expresaban dificultad para trabajar de forma cooperativa; $\mathrm{n}=8(7,1 \%)$ expresaron su satisfacción con el trabajo en equipo, comentando que habían asimilado mejor los conceptos; y $n=2(1,8 \%)$ expresaron dificultades de trabajo en equipo debido a la situación de excepcionalidad y virtualidad.

Con relación al objetivo de favorecer el aprendizaje y el rendimiento fomentando la interdependencia positiva y la responsabilidad individual, establecimos que en las pruebas grupales debería haber un mínimo de un $75 \%$ de aprobados. Los resultados nos indican que en la primera prueba de evaluación grupal aprobaron un $99,8 \%$, y en la segunda prueba aprobaron un $91 \%$.

Finalmente, respecto al objetivo de mejorar el rendimiento académico y los resultados de aprendizaje, tanto en relación a las evidencias grupales como individuales, nos planteamos que sería necesario que la media de calificaciones globales fuera superior a la de cursos académicos anteriores. Este objetivo se ha alcanzado dado que el curso anterior, a) el rendimiento fue del $72 \%$ y en este ha sido del $87 \%$ (15 puntos de diferencia absoluta, el $21 \%$ de diferencia relativa); b) el éxito fue del $75 \%$ y ahora ha sido del $93 \%$ (18 puntos de diferencia absoluta, $24 \%$ de diferencia relativa), c) la nota media ha pasado de 5,08 a 6,43 (1,35 puntos de diferencia absoluta, $27 \%$ de diferencia relativa). Estos datos se deben interpretar considerando que el indicador de rendimiento recoge el porcentaje de aprobados entre los matriculados, y que el indicador de éxito recoge el porcentaje de aprobados entre los presentados. La nota media está calculada en escala 0-10. El porcentaje de no presentados se ha mantenido constante, en un $3 \%$.

\section{Análisis Factorial Confirmatorio del CAC}

El modelo original de cinco factores no resultó estimable debido a la aparición de un caso Heywood (1931), en virtud del cual, el modelo estima variancias de correlaciones negativas o correlaciones entre variables latentes superiores a uno, no siendo ninguna de las dos cosas posibles. Este fenómeno suele darse cuando existe una alta correlación -cercana a la redundancia- entre las variables observadas. El estudio de la matriz de correlaciones entre escalas, véasela Tabla 1 , señala que esto puede deberse a la muy alta correlación entre las escalas Habilidades sociales y Procesamiento grupal.

El modelo de cuatro factores, que reúne ambas escalas (Figura 1), mostró un ajuste adecuado: (Root Mean Square Error Aproximation) RMSEA=0.065; (Standardized Root Mean Square Residual) SRMR $=0.065$; Satorra-Bentler (Satorra y Bentler, 1994) S-B $\chi 2(166)=247.049, \mathrm{p}<.001$. Los valores obtenidos para cuatro factores son similares a los reportados por los autores originales, RMSEA $=0.037$; $\mathrm{SRMR}=0.02$; Santorra-Batler B $\chi 2$ (160) $=2574.51, \mathrm{p}<001$.

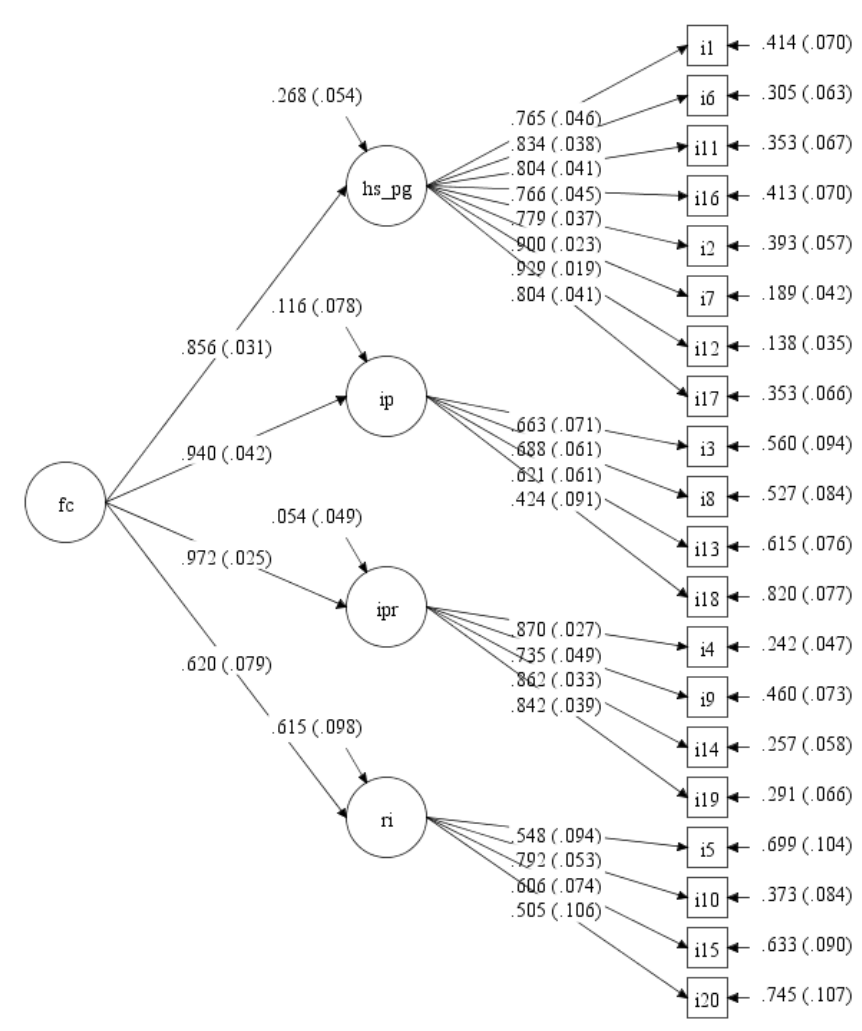

Figura 1. Modelo factorial confirmatorio (de cuatro factores) del CAC.

Nota: hs $\_g$ Habilidades sociales + Procesamiento grupal; ip $=$ Interdependencia positiva; ipr = Interacción promotora; RI = Responsabilidad social; fc $=$ Factor Cooperación

La Figura muestra, para las variables observadas, las unicidades y para las latentes, las cargas factoriales estandarizadas. Entre paréntesis, se muestran los errores estándar.

\section{CONCLUSIONES}

El objetivo principal del presente estudio fue la mejora del rendimiento académico implementando una metodología de aprendizaje cooperativo. Como objetivos específicos nos planteamos fomentar la actividad de trabajo autónomo, la asistencia a los seminarios prácticos, la interdependencia positiva y la responsabilidad individual, así como validar el $\mathrm{CAC}$ en contexto universitario. Nuestros resultados muestran que se han conseguido la mayoría de objetivos de forma satisfactoria.

Con relación al objetivo principal, señalar que se incrementó el rendimiento académico tanto a nivel grupal como a nivel individual respecto al curso académico anterior. Con relación a la actividad de trabajo autónomo, los equipos entregaron el dossier de prácticas que equivalía aproximadamente a un tercio de las horas de trabajo planificado, cumpliendo así el indicador establecido. Además, la entrega del dossier fomentó, de forma indirecta, la participación en el aula para la resolución de dudas. Sin embargo, la asistencia a los seminarios prácticos fue menor 
que el curso anterior, probablemente debido a la situación de excepcionalidad por la pandemia COVID19.

Con relación a los objetivos de fomentar la interdependencia positiva, la responsabilidad individual $\mathrm{y}$, en definitiva, el aprendizaje cooperativo, nuestros resultados muestran puntuaciones satisfactorias en el cuestionario CAC, superiores a 4 (en escala Likert 1-5), en la mayoría de las subescalas.

Asimismo, las preguntas de elaboración propia para evaluar el trabajo en equipo y los seminarios prácticos también mostraron, en general, resultados satisfactorios. En la pregunta de respuesta abierta el desacuerdo más frecuente estaba relacionado con la formación de los grupos de forma aleatoria y la gestión inadecuada de conflictos relacionados con personas que no cooperaban en el proyecto. Existen diferentes formas de generar grupos, pero permitir que el alumnado forme su propio grupo sería la opción menos indicada en este caso, pues se generan equipos homogéneos. Fomentar el aprendizaje cooperativo precisa de grupos heterogéneos con distintos componentes, intereses, perspectivas y motivaciones que promuevan un pensamiento más profundo y mayor intercambio de explicaciones (Johnson et al., 1999). Así pues, dado que el porcentaje de desacuerdo fue mínimo, y considerando las ventajas que proporciona la formación de grupos de forma aleatoria, nuestra propuesta para siguientes cursos sería mantener la misma metodología, pero facilitando a los equipos herramientas para gestionar las dificultades de tener algún participante poco colaborador. Nos planteamos, entre otras opciones, la posibilidad de que el equipo pueda "sancionar" este componente del grupo, de forma que esta persona tenga que realizar de forma individual la totalidad del proyecto (Simović, 2004).

Finalmente, y con relación a la validez estructural del CAC en el contexto universitario, nuestros resultados muestran una elevada correlación entre las escalas Procesamiento Grupal y Habilidades Sociales, a diferencia de la escala original. El modelo final estimado presenta pues cuatro subescalas, que hemos denominado como los autores originales, a excepción de la dimensión conjunta $h s p g$, habilidades sociales + procesamiento grupal. La elevada correlación entre estas dos escalas puede deberse a diversos motivos. En primer lugar, porque la muestra es bastante homogénea respecto a la edad y los intereses comunes, puesto que pertenecen a un solo curso académico y a una asignatura muy concreta. En segundo lugar, puede ser debido a un factor madurativo, pues la edad difiere considerablemente de la muestra de validación original, en un entorno de secundaria y bachillerato. Y, por último, consideramos que también puede ser debido a la dificultad derivada de la diferenciación práctica de estas dos dimensiones, ya que el factor Procesamiento Grupal implica, necesariamente, habilidades sociales tanto inter-grupales como individuales. Evaluar procesos, asimismo, es más dificultoso que evaluar un producto o bien una competencia. Probablemente evaluar procesos como el que nos ocupa precisaría de diferentes indicadores, tal y como proponen Cuéllar \& Alonso (2010) en su trabajo relacionado con la evaluación del aprendizaje cooperativo, en concreto, el proceso grupal de aprendizaje.

En definitiva, el método de aprendizaje cooperativo implementado incrementa el rendimiento académico, tal y como refieren diferentes autores (Gillies, 2014; Johnson \& Johnson, 2014; Martínez et al., 2011; Valero-García, 2017), así como también fomenta la colaboración, la interdependencia positiva y la responsabilidad individual, pero necesitamos mejores y más precisos indicadores para evaluar procesos grupales. Por otra parte, es necesario facilitar herramientas al alumnado que faciliten la gestión de los conflictos derivados de las relaciones establecidas en procesos de aprendizaje cooperativo. Así pues, basándonos en nuestros resultados consideramos que sería recomendable seguir implementado este método en siguientes cursos facilitando estas herramientas de gestión de conflictos, y evaluar el aprendizaje cooperativo mediante la aplicación del CAC en una muestra más amplia, confirmando así su validez estructural en el contexto universitario.

Como limitaciones a nuestro estudio, señalar la reducida participación del alumnado en la cumplimentación del cuestionario $\mathrm{CAC}$, probablemente debido a la baja participación en los seminarios, derivando en una muestra pequeña, bastante homogénea, y seguramente motivada por el trabajo en equipo y la cooperación. Las circunstancias sobrevenidas de la pandemia, han dificultado el proceso de implementación. Sin embargo, a pesar de estas limitaciones, consideramos que los resultados son suficientemente satisfactorios para continuar y mejorar este proyecto en los siguientes cursos académicos.

\section{AGRADECIMIENTOS}

Este proyecto de innovación docente está financiado por la Coordinación de Formación e Innovación Docente del Instituto de Ciencias de la Educación y por la Unidad de Formación y Desarrollo Profesional de la Universidad Autónoma de Barcelona (UAB), en el marco de la convocatoria competitiva anual de ayudas para proyectos de innovación y de mejora de la calidad docente.

Agradecemos a los autores del cuestionario CAC la autorización para su uso, así como también agradecemos al alumnado su participación en este trabajo.

Declaración de intereses.

Los autores declaran no tener conflicto de intereses.

\section{REFERENCIAS}

Bentler, P. M. (1990). Comparative fit indexes in structural models. Psychological Bulletin, 107(2), 238-246.

Byrne, B. M. (2012). Structural equation modeling with Mplus: Basic concepts, applications, and programming. New York: Taylor \& Francis/Routledge

Atxurra, C., Villardón-Gallego, L., \& Calvete, E. (2015). Design and validation of the cooperative learning application scale (CLAS). Revista de Psicodidactica, 20(2),

339-357. https://doi.org/10.1387/RevPsicodidact.11917

Cuéllar, A. I., \& Alonso, M. I. (2010). ¿Cómo afrontar la evaluación del aprendizaje colaborativo? Una propuesta valorando el proceso, el contenido y el producto de la actividad grupal. Revista General de Información y Documentación, 20, 221-241.

Domingo, J. (2010). El aprendizaje cooperativo y las competencias. Revista d'Innovació Docent Universitària, 2, 1-9. https://doi.org/10.1344/105.000001520

Fernández-Rio, J., Cecchini, J. A., Méndez-Giménez, A., Méndez-Alonso, D., \& Prieto, J. A. (2017). Diseño y 
validación de un cuestionario de medición del aprendizaje cooperativo en contextos educativos. Anales de Psicologia, 33(3), https://doi.org/10.6018/analesps.33.3.251321

García, M. M., López, I. G., \& Serrano, R. M. (2012). Validación del cuestionario de evaluación ACOES. Análisis del trabajo cooperativo en educación superior. Revista de Investigacion Educativa, 30(1), 87-109. https://doi.org/10.6018/rie.30.1.114091

Gillies, R. (2014). Cooperative Learning: Developments in Research. International Journal of Educational Psychology, 3(2), 125-140. https://doi.org/10.4471/ijep.2014.08

Guerra, M., Rodríguez, J., \& Artiles, J. (2019). Aprendizaje colaborativo: experiencia innovadora en el alumnado universitario. Revista de Estudios y Experiencias En Educación, 18(36), 269-281. https://doi.org/10.21703/rexe.20191836guerra5

Ibarra, M., \& Rodríguez, G. (2007). El trabajo colaborativo en las aulas universitarias: reflexiones desde la autoevaluación. Revista de Educación, 344, 335-375.

Heywood, H. B. (1931). On finite sequences of real numbers. Proceedings of the Royal Society of London. Series A, Containing Papers of a Mathematical and Physical Character 134(824), 486-501

Hu, L.-t., \& Bentler, P. M. (1999). Cutoff criteria for fit indexes in covariance structure analysis: Conventional criteria versus new alternatives. Structural Equation Modeling, 6(1), 1-55. https://doi.org/10.1080/10705519909540118

Johnson, D., \& Johnson, R. (2014). Cooperative learning in 21st Century. Anales de Psicologia, 30(3), 841-851. https://doi.org/10.6018/analesps.30.3.201241
Johnson, D., Johnson, R., \& Holubec, E. (1999). El aprendizaje cooperativo en el aula. In Paidos (Ed.), Association for Supervision and Curriculum Development.

Jöreskog, K., Sörbom, D., du Toit, S., \& du Toi, M. (2000). LISREL 8: New statistical features. Lincolnwood, IL: Scientific Software International.

Mardia, K.V. (1974) Applications of Some Measures of Multivariate Skewness and Kurtosis in Testing Normality and Robustness Studies. Sankhyā: The Indian Journal of Statistics, Series B, 36, 115-128.

Martínez, J. L., García, S. A., \& Máizquez, M. C. C. (2011). Aprendizaje cooperativo. Una experiencia en la enseñanza universitaria. Congreso Internacional de Inovación Docente.

Satorra, A., \& Bentler, P. M. (1994). Corrections to test statistics and standard errors in covariance structure analysis. In A. von Eye, \& C. C. Clogg (Eds.), Latent variables analysis: Applications for developmental research (pp. 399-419). Thousand Oaks, CA: Sage.

Simović, S. (2004). Cómo enfrentarse a los jetas y a los pasotas. $2,1-12$.

Valero-García, M. (2017). Interdependencia positiva. ReVisión, 10(1), 9-10.

Veenman, S., Van Benthum, N., Bootsma, D., Van Dieren, J., \& Van Der Kemp, N. (2002). Cooperative learning and teacher education. Teaching and Teacher Education, 18(1), 87-103. https://doi.org/10.1016/S0742051X(01)00052-X 